\title{
A FORMULA FOR AN EQUIVALENT INVARIANT MEASURE
}

\author{
DOROTHY MAHARAM
}

\begin{abstract}
Let $T$ be a nonsingular 1-1 measurable transformation of a finite measure space $(X, \mathscr{B}, m)$. A simple construction is given for a $\sigma$-finite measure $\mu$, equivalent to $m$ and invariant under $T$, when such a measure exists.
\end{abstract}

1. Introduction. In [1] the author gave necessary and sufficient conditions for the existence of a $\sigma$-finite invariant measure $\mu$ for a nonsingular 1-1 measurable transformation $T$ of a measure space $(X, \mathscr{B}, m)$ onto itself, where $\mu$ is required to be equivalent to $m$, and where (without real loss of generality but with considerable simplification of details) $m$ is assumed finite. My first proof that the conditions are sufficient was long and complicated; the much better proof in [1] (kindly supplied by Y. Ito) is indirect. Here we give an even simpler proof, which provides a simple explicit formula for such an invariant measure (which, of course, need not be unique in general). We recall the following notation from [1].

The Radon-Nikodym derivative of $T^{n}(n \in \mathbf{N}=\{0,1,2, \ldots\})$ is $\omega_{n}$, so $m\left(T^{n} B\right)=$ $\int_{B} \omega_{n}(x) d m(x)$ for all $B \in \mathscr{B}$. We may (and do) freely discard invariant null sets from $X$, and thus arrange that $0<\omega_{n}(x)<\infty$ for all $x \in X$ and $n \in \mathbf{N}$. For all $\alpha, \beta \geqslant 0$, the "relative density" $d(\alpha, \beta, x)$, here renamed $\delta(\alpha, \beta, x)$, is

$$
\lim _{k \rightarrow \infty} \frac{\mid\left\{n \in \mathbf{N}: n \leqslant k \text { and } \omega_{n}(x) \geqslant \alpha\right\} \mid}{\mid\left\{n \in \mathbf{N}: n \leqslant k \text { and } \omega_{n}(x) \geqslant \beta\right\} \mid} ;
$$

this limit exists (possibly infinite) for all $\alpha, \beta \geqslant 0$ and (almost) all $x$. We put

$$
I_{\beta}(x)=\int_{0}^{\infty} \delta(\alpha, \beta, x) d \alpha,
$$

and have that $\beta \leqslant I_{\beta}(x) \leqslant \infty ; I_{\beta}(x)$ increases with $\beta$ (for fixed $x$ ); and (an observation I owe to A. H. Stone)

$$
\int_{0}^{\infty} \frac{d \beta}{I_{\beta}(x)}=0 \text { or } 1 \text { for (almost) all } x .
$$

(See [1, p. 226].)

To avoid repetition the term "invariant measure" in what follows means " $\sigma$-finite measure, equivalent to $m$, and invariant under $T$ ".

Received by the editors February 8, 1984

1980 Mathematics Subject Classification. Primary 28D05; Secondary 58F11.

Key words and phrases. Invariant measure.

(C)1985 American Mathematical Society $0002-9939 / 85 \$ 1.00+\$ .25$ per page 
The main theorem of [1] asserts that an invariant measure exists if, and only if,

$$
\int_{0}^{\infty} \frac{d \beta}{I_{\beta}(x)}=1 \text { a.e. }
$$

(For some other equivalent conditions, see [1, Theorem 4].)

2. The proof of "only if" in [1] is relatively straightforward; here we re-prove the converse by deriving a simple formula for a suitable measure, assuming that (1) holds.

First we note that (whether or not (1) holds) we have $\omega_{n}(x)=\omega(x) \omega_{n-1}(T x)$ (where $\omega(x)$ is written for $\omega_{1}(x)$ ), and thus

$$
\begin{aligned}
\delta(\alpha, \beta, x) & =\lim _{k \rightarrow \infty} \frac{\mid\left\{n \in \mathbf{N}: n \leqslant k \text { and } \omega_{n-1}(T x) \geqslant \alpha / \omega(x)\right\} \mid}{\mid\left\{n \in \mathbf{N}: n \leqslant k \text { and } \omega_{n-1}(T x) \geqslant \beta / \omega(x)\right\} \mid} \\
& =\delta(\alpha / \omega(x), \beta / \omega(x), T x),
\end{aligned}
$$

whence a simple calculation gives

$$
I_{\beta}(x)=\omega(x) I_{\beta / \omega(x)}(T x),
$$

and so, if $b \geqslant 0$,

$$
\int_{0}^{b} \frac{d \beta}{I_{\beta}(x)}=\int_{0}^{b / \omega(x)} \frac{d \beta}{I_{\beta}(T x)} .
$$

Now assume (1), and fix $x$ for the present. If $I_{\beta}(x)$ becomes infinite for some $\beta>0$, put $\beta^{*}\left(=\beta^{*}(x)\right)=\inf \left\{\beta: I_{\beta}(x)=\infty\right\}$; then the function, defined for $b \geqslant 0$ by

$$
b \mapsto \int_{0}^{b} \frac{d \beta}{I_{\beta}(x)}
$$

is continuous, nondecreasing, and strictly increasing for $0 \leqslant b \leqslant \beta^{*}$, and takes the value 1 at $b=\beta^{*}$. Thus there is a unique $b(x)>0$ such that

$$
\int_{0}^{b(x)} \frac{d \beta}{I_{\beta}(x)}=\frac{1}{2}
$$

If $I_{\beta}(x)$ remains finite for all (finite) $\beta>0$, similar, but simpler, reasoning again gives a unique $b(x)>0$ satisfying (3).

Now from (2) we have

$$
\int_{0}^{b(x) / \omega(x)} \frac{d \beta}{I_{\beta}(T x)}=\frac{1}{2}
$$

so

$$
b(x) / \omega(x)=b(T x) .
$$

Also, $b(x)$ is a measurable function of $x$, for $\{x: b(x)>c\}$ is precisely the set of $x$ 's for which $\int_{0}^{c} d \beta / I_{\beta}(x)<1 / 2$; and this is measurable for each $c$, by Fubini's theorem. 
Thus (4) (by a familiar and elementary calculation) shows that the measure $\mu$ defined by

$$
\mu(B)=\int_{B} b(x) d m(x) \quad(B \in \mathscr{B})
$$

is $T$-invariant, as required.

3. Remarks. (i) Even if (1) does not hold, the set of $x$ 's for which $\int_{0}^{\infty} d \beta / I_{\beta}(x)=1$ is the largest subset of $X$ (modulo null sets) on which an invariant measure exists, and the present construction, restricted to this subset, gives an invariant measure on it.

(ii) When (1) holds we can obtain all ( $\sigma$-finite, $m$-equivalent) $T$-invariant measures by replacing the constant value $1 / 2$ in the definition of $b(x)$ by an arbitrary measurable invariant function $f$ with values in $(0,1)$.

(iii) Necessary and sufficient conditions for the existence of a finite invariant measure, and simple formulae for one when it exists, are well known; see for example [1, Theorem 1].

\section{REFERENCES}

1. Dorothy Maharam, Invariant measures and Radon-Nikodym derivatives, Trans. Amer. Math. Soc. 135 (1969), 223-248.

DePartment of Mathematics, University of Rochester, Rochester, New York 14627 